\section{OC8 HIGH PREVALENCE OF WT1 GENE MUTATIONS IN PATIENTS WITH STEROID-RESISTANT NEPHROTIC SYNDROME}

Dmitry Sladkov*, Kirill Savostyanov, Alexey Nikitin, Natalya Zhurkova, Alexander Pakhomov, Anastasia Rykunova, Peter Anan'in, Alexey Tsygin. Federal State Autonomous Institution 'National Medical Research Center for Children's Health' of the Russian Federation Ministry of Health, Moscow, Russian Federation

\subsection{6/archdischild-2019-epa.8}

Background and aims Steroid-resistant nephrotic syndrome (SRNS) is the rare disease and holds a special place in structure of nephrotic syndrome in children, it takes up to $15-25 \%$ among other forms of the nephrotic syndrome. SRNS is one of the most common causes of end-stage kidney failure. The majority of SRNS cases are caused by genetic mutations leading to structural and functional glomerular injuries. Treatment strategy in children with SRNS is defined by results of molecular genetic testing and morphological diagnosis after kidney biopsy. Research is focused on identification of pathogenic gene variants leading to kidneys hereditary diseases development united by SRNS symptoms complex.

Methods The research included 83 patients (40 girls, 43 boys) aged from 0 up to 16 years with SRNS who undergone molecular genetic testing by next generation sequencing (NGS) with further results validation by Sanger sequencing and bioinformatics analysis by means of the program Alamut.

Results Mutations in WT1 gene were revealed in 13 cases (10 girls, 3 boys, mean age $-7,5$ years, manifestation mean age -2 years), four patients have congenital nephrotic syndrome. Revealed morphological variants: focal segmental glomerulosclerosis in 10 cases, minimal changes disease - 3 cases. Pathogenic variants of WT1 gene: 6 missense (c.1217G >A, p.R406K; c.1270A>G, p.K424E; c.1311T>G, p.F437L; c.1399C>T, p.R467W; c.1407C>A, p.D469E; c.1433A>G, p.H478R) and 2 splicing (c.1447+4C>T, c.1447 $+5 G>A$ ) mutations (NM 024426.5 transcript). We revealed two novel variants (c.12 $17 G>A, \quad$.R $406 K ; c .1311 T>G, p$. F437L) undescribed in HGMD professional database. The most common mutation in WT1 gene is c. $1447+5 G>A$. Mutations in WT1 gene cause various clinical phenotypes: Denys-Drash syndrome (8\%), Frasier syndrome (15\%), isolated SRNS (77\%). All patients with WT1 mutations have significantly lower levels of proteinuria against whole patient cohort, nearly all have sustained hypertension and intermittent hematuria.

Conclusion It was established that in $16 \%$ of all investigated cases the key factors for the manifestation and progression of SRNS are WT1 gene mutations. Mutations c.1399C>T and c. $1433 A>G$, are associated with rapid progression and early end-stage kidney failure in the age under 6 years.

\section{OC9 IMPACT OF COMBINED ANTIRETROVIRAL PROPHYLAXIS ON HEALTH OUTCOMES IN HIV-EXPOSED NEONATES}

${ }^{1}$ Alla Petrova, ${ }^{1}$ Anastasiya Vanyarkina*, ${ }^{2} J u l i a$ Plotnikova, 'Lyubov Rychkova, 'Ekaterina Moskaleva. 'Scientific Centre for Family Health and Human Reproduction Problems, Irkutsk, Russian Federation; ${ }^{2}$ Regional Centre on Prevention and Control of AIDS, Irkutsk, Russian Federation

10.1136/archdischild-2019-epa.9
Modern approaches to administration of antiretroviral therapy (ART) to prevent HIV transmission from an infected woman to her child during pregnancy, labor, and delivery (in case she does not breastfeed her child) significantly reduce the risk of newborn infection. Combined ART is indicated for children with perinatal HIV exposure at high risk of being infected and includes administration of three oral antiretrovirals: zidovudine, nevirapine, lamivudine.

The aim To assess the health of newborns, who underwent the combined antiretroviral therapy to prevent perinatal HIV transmission.

Materials and methods We conducted a post-hoc analysis of 163 cases of HIV-positive women and their newborns at Irkutsk Perinatal Centre (tertiary medical centre), covering 2017-2018 years. Inclusion criteria were high risk of perinatal HIV transmission (a woman did not take antepartum ART, unknown HIV viral load or viral load near delivery is more than 50 copies $/ \mathrm{ml}$ ), a positive result of rapid (point-of-care) HIV antibody test in the mother during delivery, and epidemiological parameters. All newborns with perinatal HIV exposure were given three drug regimen, were formula-fed, and their blood was tested by means of polymerase chain reaction. The follow-up of newborns included clinical monitoring, assessment of hematologic (anemia, neutropenia) and other adverse effects of ART.

Results Out of 445 newborns with perinatal HIV exposure, 163 (36.6\%) neonates underwent combined ART. The most frequent causes of combination ART prescription were: no adherence $(34.8 \%)$ to or no taking (39\%) ART by the mother of the neonate during pregnancy, high HIV viral load (17.2\%), and epidemiological indications (parenteral substances use during pregnancy - 9\%). Fifty-six (34.3\%) cases resulted in premature births. An average HIV viral load near delivery was $2800[150 ; 8100]$ copies $/ \mathrm{ml}$. Average body weight of newborns was 2590 [700; 4180] grams. Among adverse clinical effects of ART in $60 \%$ of prematurely-born HIV-exposed neonates, symptoms of enteropathy prevailed; $19.7 \%$ of newborns developed severe anemia by the $28^{\text {th }}$ day after birth. HIV RNA was detected in $1(0.6 \%)$ newborn of this group. Four (2.4\%) newborns with perinatal HIV exposure died.

Conclusions Lack of social and medical support of reproductive women, belonging to risk groups, increases the risk of perinatal HIV transmission. Personalized approach to ART planning helps not only to reduce the risk of mother-to-child transmission, but also avoid toxic side-effects of preparations on neonates with perinatal HIV exposure.

\section{OC10 I HAVE A DEAD PERSON'S KIDNEY IN ME': THE STRUGGLES OF SELF-IDENTITY AMONG ADOLESCENT KIDNEY RECIPIENTS IN IRELAND}

${ }^{1}$ Colleen O'Neill*, ${ }^{1}$ Briege Casey, 'Veronica Lambert, ${ }^{2}$ Atif Awan. 'Dublin City University, Dublin, Ireland; ${ }^{2}$ Temple Street Children's University Hospital, Dublin, Ireland

\subsection{6/archdischild-2019-epa.10}

Objective Chronic Kidney Disease (CKD) is a complex disease that requires lifelong treatment. Transplantation is the goal standard treatment, however; it is not without its challenges. While previous research has demonstrated that transplantation increases survival rates and enhances young people's quality of life, little is known about the impact of kidney 